\title{
Alamouti Distributed Space-Time Coding with Relay Selection
}

\author{
Dokheyl M. Al-Qahtani \\ Electrical Engineering Department \\ King Saud University \\ Riyadh 12372, Saudi Arabia \\ Email: e.dkil@hotmail.com
}

\author{
Abdulhameed M. Al-Sanie \\ Electrical Engineering Department \\ King Saud University \\ Riyadh 12372, Saudi Arabia \\ Email: sanie@ksu.edu.sa
}

\begin{abstract}
This paper examines the impact of relay selection on the performance of Alamouti distributed orthogonal spacetime block coding (Al-DSTC) in a two-relay network. The SNRmaximizing is implemented at the receiver based on selecting the relay to cooperate or not. The receiver can always find the optimal selected relay by exhaustive search where the number of relays is only two. It is shown in this work that the proposed scheme obtains full diversity order and provides an additional array gain compared to conventional Al-DSTC. The proposed scheme has a lower bit error rate if compared to the beamforming when the transmit power is high. Our results show that the proposed scheme is more power efficient than the conventional Al-DSTC and cooperative beamforming. Also, the proposed scheme increase life time of the relay since $50 \%$ of the time the relay is off.
\end{abstract}

Index Terms-Relay Selection, Alamouti distributed orthogonal space-time block coding, Amplify-and-Forward, Wireless relay networks.

\section{INTRODUCTION}

The wireless communication channel suffers from noise, interference and multipath fading [1], [2]. One of the most powerful techniques to achieve reliable communication over a wireless channel is a diversity which ensures that more than one copy of the transmitted signal arrives at the receiver (Rx) on statistically independent channels [3]. According to the domain where diversity is introduced, diversity techniques are classified into time [4], frequency [5] and spatial [6] diversity. Cooperative diversity has been recently shown to be a promising method to achieve spatial diversity in wireless networks. In a wireless network, independent channels between the transmitter and receiver exist when the multiple users in the network work as relays for each other. Cooperative diversity achieves the significant benefit of diversity gain as in traditional MIMO systems without requiring multiple antennas at individual nodes.

Among the most widely used relaying techniques are decode-and-forward (DF) [7]-[9] and amplify-and-forward (AF) [10]-[12]. Clearly, AF relaying has implementation advantages over DF relaying because no channel estimation nor decoding is required at $\mathrm{AF}$ operating relays. In practice, multiple relays can be allocated to help the communication. Then, more than one relay can be assigned for forwarding the information to the destination. A variety of cooperative protocols have been proposed in the literature with different level of channel state information (CSI) at the relays [12]-[19]. When channel state information (CSI) of a communication link is available at the relay nodes cooperative beamforming have been proposed [13]-[17]. In cooperative beamforming, each relay precompensate the channel effects and adjusts their transmitting power before transmission to maximize signal-tonoise ratio (SNR) at the receiver. Cooperative beamforming is studied in [13] under total relay power constraint, and in [16], under individual relay power constraints. In [17], the authors proposed cooperative beamforming by selecting the cooperating relays globally for networks with simple relays and complexity constraints, in which the arbitrary power adjustment may not be practical or desirable. When channel information is not available at the relay nodes, a distributed space time codes (D-STC) was proposed, which achieves a diversity factor of $R$ in a $R$-relay wireless network. This method does not require decoding at the relays and for high SNR it achieves the optimal diversity factor [19]. Design of practical D-STC that lead to reliable communication in wireless relay networks has also been recently considered [18], [20], [21] . In [18], practical distributed space-time codes were proposed using orthogonal and quasi-orthogonal designs which are originally used in the design of spacetime codes for multiple-antenna systems. It is well known that orthogonal space-time block codes decrease the complexity of the decoder where each symbol is decoded separately. Furthermore, a distributed orthogonal space-time block code (D-OSTBC) can easily apply in a wireless network since the entries of codewords are linear in the information symbols and their conjugates. However, D-OSTBC has scale free property, meaning that it still offers the maximum possible diversity even if some of the relays are not used. Alamouti distributed orthogonal space-time block code (Al-DSTC) is the simplest form of D-OSTBC by using two relay in a wireless network. Al-DSTC is the only D-OSTBC that achieves full rate code. In the conventional D-OSTBC every relay cooperates and each relay transmits with fixed power (e.g., [12], [18], [19]).

Recently, there are many schemes that combine D-STBC with adaptive relay power allocation [22]-[24]. In [22], under full knowledge of channel state information at the relay, power allocation uses maximum SNR subject to the fixed transmit 
power during the second phase led to opportunistic relaying. Furthermore, they proposed two power allocation methods based on bit error rate (BER) minimization under statistical channel state information. The authors in [23] showed that the optimal power allocation based on maximizing the SNR with full CSI at the relay, may fail to distribute the power to all the relays, and thus compromise the diversity of the code. Then they proposed a power allocation scheme that minimizing the "amount of fading" based on statistical CSI at the relay. In [24], analytically find the optimal relay power allocation that minimizes an upper bound on the pairwise error probability (PEP) under statistical CSI. Unlike the conventional D-STBC systems, a feedback channel is required for D-STBC system with adaptive relay power allocation in order to notify CSI to the relay.

Besides, we can improve the performance of D-STBC designed in a network with $R$ relays by using binary power allocation (selecting the cooperating relays). Binary power allocation offers two main benefits over continuous adaptive power allocation: (i) Simple nodes design. (ii) The amount of overhead and feedback exchange among network nodes is significantly reduced. Each node in the network can transmit its signals using one of the two power levels: zero and its maximum power. Since in this case a relay either cooperates with its full power or remains silent, the problem is how to select the cooperating relays.

In this paper, we combine Al-DSTC with relay selection (RS) in a two-relay wireless networks to obtain full diversity order and provide an additional array gain compared to conventional Al-DSTC. The selection criterion was based on the maximizing received signal-to-noise ratio. The main contributions of this paper are:

1) Exploiting the Alamouti distributed orthogonal spacetime block code to:

a) Achieve high spectral efficiency (full rate code).

b) Provide a full diversity order in a two-relay wireless networks when the channel state information (CSI) is not available at the relays (Simple Relay).

c) Decrease the complexity of the decoder (Each symbol is decoded separately using only linear processing).

d) Easily applied in a wireless network as the entries of codewords are linear in the information symbols and there conjugates.

e) Get scale-free property, meaning that it still offers the maximum possible diversity even if some of the relays are not used.

2) Exploiting the Relay Selection (RS) to:

a) Reduce the amount of overhead and feedback exchange among network nodes.

b) Reduce the complexity of relay node since the selection algorithim is done at the receiver and each node can transmit with one of the two power levels: zero and its maximum power.

c) Increase the array gain compared to the conven-
TABLE I

LIST OF KEY PARAMETERS AND VARIABLES

\begin{tabular}{|c||c|}
\hline \hline Parameters & Description \\
\hline \hline$R$ & Number of relays employed \\
\hline$T$ & Coherence interval \\
\hline$f_{i}$ & Channel from the transmitter to the $i$ th relay \\
\hline$g_{i}$ & Transmit signal vector at the transmitter \\
\hline $\mathbf{s}$ & Received signal vector at the $i$ th Relay \\
\hline $\mathbf{r}_{i}$ & Additive noise vector at the $i$ th Relay \\
\hline $\mathbf{v}_{i}$ & Additive noise vector at the receiver \\
\hline $\mathbf{n}$ & Transmit signal vector at the $i$ th Relay \\
\hline $\mathbf{t}_{i}$ & Transmitted space time codeword \\
\hline $\mathbf{S}$ & Equivalent channel of the system \\
\hline $\mathbf{h}$ & Equivalent noise of the system \\
\hline $\mathbf{w}$ & Covariance matrix of $\mathbf{w}$ \\
\hline $\mathbf{R}_{\mathbf{W}}$ & Average power per transmission in the $1^{\text {st }}$ step \\
\hline$P_{1}$ & Average power per transmission used at every relay \\
\hline$P_{2}$ &
\end{tabular}

tional Al-DSTC without relay selection.

d) Increase the life time of the relay nodes.

The outline of this paper is organized as follows. In Section I, we provide a mathematical model for the two relay wireless networks. In Section III, briefly explain Alamouti distributed orthogonal space-time block coding. In Section IV, we describe the Maximum likelihood (ML) detection and analyze received SNR for Alamouti distributed orthogonal space-time block coding. In Section V, we investigate the selection criteria and the optimal relay selection algorithm. Simulated performance of the proposed system is provided in Section VI. Finally, Section VII, presents concluding remarks.

Notation We denote scalars by lower case letters, e.g. $w$, vectors by lower case bold letters, e.g. w, and matrices with upper case bold face letters, e.g. W. We use $\mathbf{w}_{i}$ to denote the $i$ th column of matrix $\mathbf{W}$ and $w_{j}$ to denote the $j$ th entry of vector $\mathbf{w}$. We use $\mathbf{I}_{N}$ to denote the $N \times N$ identity matrix and $\mathbf{O}_{N, M}$ to denote an $N \times M$ zero matrix. In addition, $\operatorname{Diag}(\mathbf{w})$ denotes the diagonal matrix with diagonal elements given by the vector w. Finally, we use $(.)^{t},(.)^{*}$ and $(.)^{H}$ to respectively denote the transpose, conjugate and Hermitian operations.

For the convenience of the reader, we have summarized the key system parameters and variables used in the paper in Table I.

\section{SySTEM MODEL}

Fig. 1 illustrates a wireless network model, in which one transmit-and-receive pair and two parallel AF relays are employed. Every node is equipped with a single antenna that can be used for both transmission and reception. It is assumed that all nodes operate in half-duplex mode, i.e., they cannot transmit and receive simultaneously in the same frequency band. There is no direct link from the transmitter to the receiver. Also, it is assumed that no information is exchanged between the relay nodes. In this set-up, the relays cooperate 


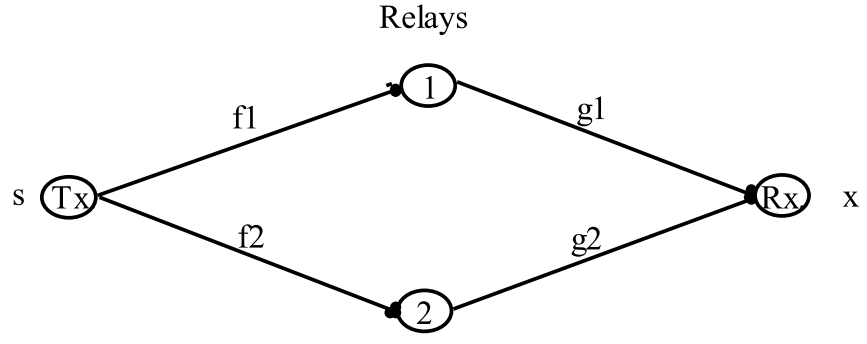

Fig. 1. Network System Model

with each other to form an Alamouti distributed orthogonal space-time block codeword at the receiver. Denote the channel from the transmitter to the $i$ th relay and the channel from the $i$ th relay to the receiver as $f_{i}$ and $g_{i}$, respectively. We assume Rayleigh fading channels: $f_{i}$ and $g_{i}$ are independent and identically distributed (i.i.d.) zero-mean and unit-variance complex Gaussian random variables. We use a block-fading model by assuming a coherence interval $T=2$, that is, $f_{i}$ and $g_{i}$ keep constant for a block of two transmissions and jump to other independent values for another block. Also, the background noise at the relays and receiver is assumed to be i.i.d. additive white Gaussian noise (AWGN) with zero-mean and unit-variance.

Assume that the CSI of a communication link is available at its receiver but not at its transmitter, i.e., assume channel state information at the receiver (CSIR only). More specically, $i$ th relay knows $f_{i}$, and the receiver knows all channels $f_{1}, f_{2}$ and $g_{1}, g_{2}$. With no forward channel information at relays, Al-DSTC [18] is chosen to be the cooperative scheme to exploit the diversity in a two relay wireless network. Al-DSTC uses the idea of distributed linear dispersion space-time codes [19]. With the help of limited feedback, we aim to improve the network performance by combining Al-DSTC with relay selection.

A two-step AF protocol is used in the network to send information, as depicted in Fig. 1. Information bits are encoded into the vector $\mathbf{s}=\left[s_{1}, s_{2}\right]^{t}$ with the normalization $E\left\{\mathbf{s}^{H} \mathbf{s}\right\}=1 . E$ indicates the expectation of a random variable. During the first step, the transmitter sends $\sqrt{2 P_{1}}$ s over two symbol intervals. The received signal at the $i$ th relay can be written as:

$$
\mathbf{r}_{i}=\sqrt{2 P_{1}} f_{i} \mathbf{s}+\mathbf{v}_{i}
$$

Each relay linearly transform their received signals to obtain $\mathbf{t}_{i}$ which sends from $i$ th relay during the second step over two symbol intervals. The received signal at the receiver can be written as:

$$
\mathbf{x}=\sum_{i=1}^{2} g_{i} \mathbf{t}_{i}+\mathbf{n} .
$$

\section{Alamouti Distributed orthogonal SPACE-TIME BLOCK CODE}

The transmit signal at the $i$ th relay is designed to be a linear function of its received signal and its conjugate

$$
\begin{aligned}
\mathbf{t}_{i}= & \sqrt{\alpha_{i} P_{R_{i}}}\left(\mathbf{A}_{i} \mathbf{r}_{i}+\mathbf{B}_{i} \overline{\mathbf{r}}_{i}\right) \\
= & \sqrt{2 \alpha_{i} P_{1} P_{R_{i}}}\left(f_{i} \mathbf{A}_{i} \mathbf{s}+f_{i}^{*} \mathbf{B}_{i} \overline{\mathbf{s}}\right) \\
& +\sqrt{\alpha_{i} P_{R_{i}}}\left(\mathbf{A}_{i} \mathbf{v}_{i}+\mathbf{B}_{i} \overline{\mathbf{v}}_{i}\right)
\end{aligned}
$$

where $\mathbf{A}_{i}$ and $\mathbf{B}_{i}$ are $T \times T$ complex matrices that generate orthogonal space time code at the receiver. $P_{R_{i}}$ denotes the amplifier power of the $i$ th relay given by

$$
P_{R_{i}}= \begin{cases}\frac{P_{2}}{\left(P_{1}\left|f_{i}\right|^{2}+1\right)} & \text { short-term } \\ \frac{P_{2}}{\left(P_{1}+1\right)} & \text { long-term }\end{cases}
$$

Compared to the conventional DSTC proposed in [18], [19], this design introduces the positive coefcients $\alpha_{1}, \alpha_{2}$ to control the relay transmit powers. If the $i$ th relay with full power then $\alpha_{i}=1$ where if the $i$ th relay not cooperate $\alpha_{i}=0$.

In this paper, we consider the special case when either $\mathbf{A}_{i}=$ $\mathbf{O}_{2,2}, \mathbf{B}_{i}$ is unitary or $\mathbf{B}_{i}=\mathbf{O}_{2,2}, \mathbf{A}_{i}$ is unitary. Define

$$
\left\{\begin{array}{l}
\hat{\mathbf{A}}_{i}=\mathbf{A}_{i}, \hat{f}_{i}=f_{i}, \hat{\mathbf{v}}_{i}=\mathbf{v}_{i}, \mathbf{s}^{(i)}=\mathbf{s}, \quad \text { if } \mathbf{B}_{i}=\mathbf{O}_{2,2} \\
\hat{\mathbf{A}}_{i}=\mathbf{B}_{i}, \hat{f}_{i}=f_{i}^{*}, \hat{\mathbf{v}}_{i}=\mathbf{v}_{i}{ }^{*}, \mathbf{s}^{(i)}=\mathbf{s}^{*}, \quad \text { if } \mathbf{A}_{i}=\mathbf{O}_{2,2}
\end{array}\right.
$$

From (4), we have

$$
\mathbf{t}_{i}=\sqrt{2 \alpha_{i} P_{1} P_{R_{i}}} \hat{f}_{i} \hat{\mathbf{A}}_{i} \mathbf{s}^{(i)}+\sqrt{\alpha_{i} P_{R_{i}}} \hat{\mathbf{A}}_{i} \hat{\mathbf{v}}_{i} .
$$

Then, the received signal at the receiver can be

$$
\mathbf{x}=\sqrt{2 P_{1}} \mathbf{S Q P h}+\mathbf{w}
$$

where

$$
\begin{gathered}
\mathbf{S}=\left[\hat{\mathbf{A}}_{1} \mathbf{s}^{(1)}, \hat{\mathbf{A}}_{2} \mathbf{s}^{(2)}\right] \\
\mathbf{h}=\left[\hat{f}_{1} g_{1}, \hat{f}_{2} g_{2}\right]^{t}
\end{gathered}
$$

and

$$
\mathbf{w}=\sum_{i=1}^{2} \sqrt{\alpha_{i} P_{R_{i}}} g_{i} \hat{A}_{i} \hat{\mathbf{v}}_{i}+\mathbf{n}
$$

Therefore, without decoding, the relays generate a spacetime codeword $S$ distributively at the receiver. $\mathbf{Q}=\operatorname{Diag}(\boldsymbol{\alpha})$ is the selection precoding matrix where $\boldsymbol{\alpha}=\left[\alpha_{1}, \alpha_{2}\right]^{t}$ is the selection vector. Thus, the relay selection affects the system equation through a diagonal precoding matrix. $\mathbf{P}=\operatorname{Diag}(\mathbf{p})$ is the amplifier power matrix where $\mathbf{p}=\left[\sqrt{P_{R_{1}}}, \sqrt{P_{R_{2}}}\right]^{t}$ is the amplifier power vector. The Matrix $S$ in (6) should be properly designed to ensure full-rate full-diversity Alamouti distributed orthogonal space-time block coding. Design the matrices used at the two relays as

$$
A_{1}=I_{2}, B_{1}=O_{2}, A_{2}=O_{2}, B_{2}=\left[\begin{array}{cc}
0 & -1 \\
1 & 0
\end{array}\right]
$$

The space-time code word formed at the receiver has the following form: 


$$
\left[\begin{array}{cc}
s_{1} & -s_{2}^{*} \\
s_{2} & s_{1}^{*}
\end{array}\right]
$$

\section{ML DeCODING AND SNR ANALYSIS}

Since $g_{i}, \mathbf{v}_{i}$ and $\mathbf{n}$ are complex Gaussian random variables, which are jointly independent, it is straightforward to prove that the equivalent noise $\mathbf{w}$ is a complex Gaussian random vector when $g_{i}$ are known, whose mean is 0 and covariance matrix is

$$
\begin{aligned}
\mathbf{R}_{\mathbf{w}} & =\mathbf{I}_{2}+\sum_{i=1}^{2} \alpha_{i} P_{R_{i}}\left|g_{i}\right|^{2} \hat{\mathbf{A}}_{i} \hat{\mathbf{A}}_{i}^{H} \\
& =\left(1+\sum_{i=1}^{2} \alpha_{i} P_{R_{i}}\left|g_{i}\right|^{2}\right) \mathbf{I}_{2}
\end{aligned}
$$

Since the covariance matrix is a multiple of the identity matrix, maximum likelihood decoding of Al-DSTC can be achieved using only linear processing at the receiver which decouple the information symbols. The SNR at the receiver for each symbol is given by:

$$
S N R=\frac{2 P_{1} \sum_{i=1}^{2} \alpha_{i} P_{R_{i}}\left|f_{i}\right|^{2}\left|g_{i}\right|^{2}}{\left(1+\sum_{i=1}^{2} \alpha_{i} P_{R_{i}}\left|g_{i}\right|^{2}\right)}
$$

\section{RELAY SELECTION ALGORITHIM}

In this section we describe the proposed relays selection algorithm that we employ to improve the performance of the Alamouti distributed orthogonal space-time block code scheme. The optimal relay is selected to maximize the signalto-noise ratio (SNR) at the receiver. The main problem is

$$
\begin{array}{ll}
\max _{\alpha_{1}, \alpha_{2}} & S N R \\
\text { subject to } & \alpha_{i} \in\{0,1\}
\end{array}
$$

With perfect knowledge of all channels $f_{1}, f_{2}$ and $g_{1}, g_{2}$ at the receiver, it can select a subset of relays that maximizes the received SNR. The relay selection algorithm is divided into the following steps:

1) The receiver calculates SNR for all possible different selection of relay.

2) The receiver finds the maximum SNR and returns the relay selection coefficients to the relay.

3) Each selected relay transmits an associated column of the underlying Alamouti matrix.

\section{Simulation Results}

In this section, we show the simulated bit error rate (BER) of the proposed system. We assume an equal power constraint on both the transmitter and the relays, i.e. $P_{1}=P_{2}=P$. In all figures, the horizontal axis indicates $P$. All channels are generated as i.i.d. complex Gaussian random variables with zero-mean and unit-variance. The noises at the relays and receiver are also i.i.d. complex Gaussian random variables with zero-mean and unit-variance.

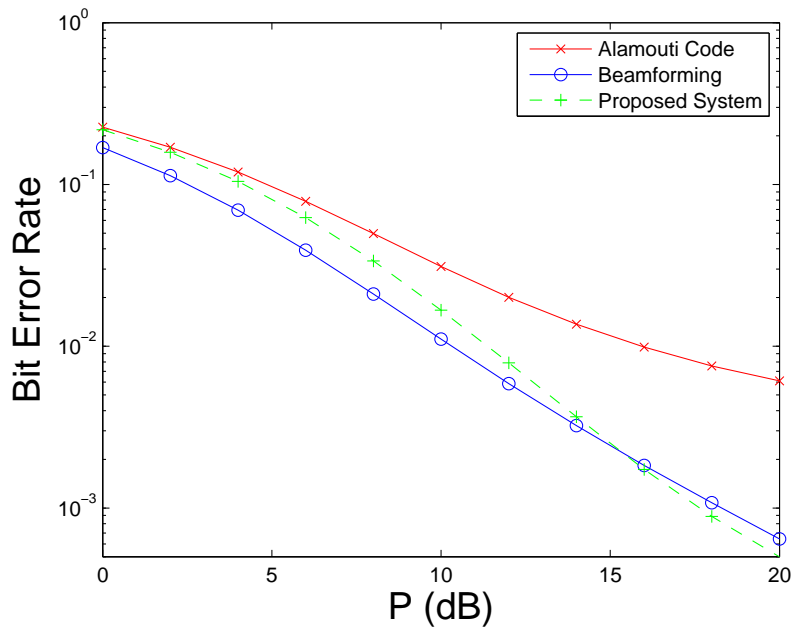

Fig. 2. BER performance.

In Fig. 2, simulated bit error rate of the proposed scheme are compared to conventional Alamouti Distributed space time code, and to cooperative beamforming in a two-relay network. In cooperative beamforming each relay precompensate the channel phase effects before transmission so that the signals constructively combine at the receiver. Coding gain is visible through the parallel shift of the BER curves of the proposed scheme compared to the conventional Al-DSTC BER curve. The proposed scheme has lower BER compared to the beamforming when the transmit power is high. The proposed system has lower BER compared to the conventional Al-DSTC for all P.

Since, the power used in the second step of the transmission is not same for this three schemes, making it difficult to have a fair comparison. In order to see a fair comparison between the scheme, we compare the performance of this scheme based on the power efficiency. Power efficiency is given by [17]

$$
\begin{aligned}
P_{e} & =\frac{S N R}{\text { Total power per symbol transmission }} \\
& =\frac{S N R}{P_{1}+\left(\alpha_{1}+\alpha_{2}\right) P_{2}}
\end{aligned}
$$

Where for conventional Distributed Alamouti space time code and cooperative beamforming $\alpha_{1}=\alpha_{2}=1$ because all relay cooperates in the second step of transmission.

Fig. 3, shows the power efficiency comparison of the three schemes at BER simulated in Fig. 2. We found that the proposed scheme is more efficient than the conventional Distributed Alamouti space time code and cooperative beamforming.

In Fig. 4, the percentage of the average number of relay selected in proposed system where BER of this system simulated in Fig. 2. The proposed scheme uses $50 \%$ of time only one relay which save the power in the 2 nd step of transmission. The proposed system increases the life time of the relay since 


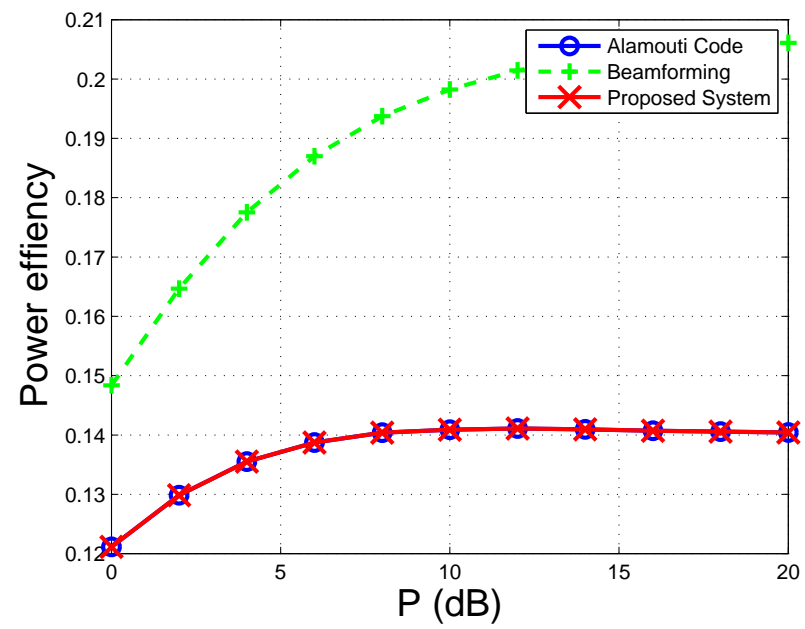

Fig. 3. Power efficiency.

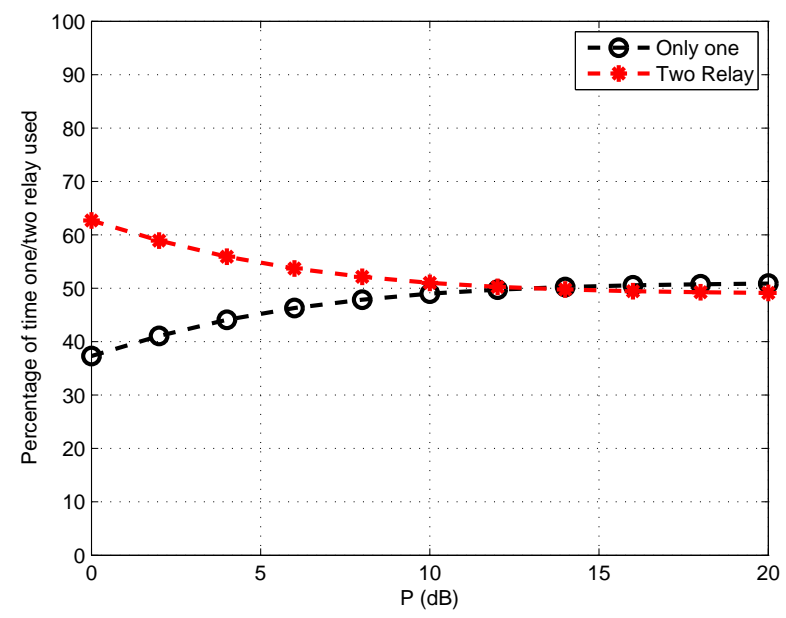

Fig. 4. Percentage of time one/two relay used.

$50 \%$ of time the relay is off.

\section{CONCLUSION}

In this paper, we proposed a novel technique that combines Al-DSTC with relay selection in a two-relay networks to obtain full diversity order and provide an additional array gain. The scheme is based on a two-step amplify and forward DSTC cooperative protocol. The key idea in developing this technique is exploiting one bit feedback for each relay indicating whether it cooperates or not. The optimal relay is selected to maximize the signal-to-noise ratio (SNR) at the receiver. Since the receiver knows all the channels, it can always find the optimal selected relay by exhaustive search. The relay selection algorithm complexity should not be a problem in this scheme where the number of relays is only two. Simulated BER performance shows that the proposed scheme preserves full-diversity order and it also provides largely additional array gain compared to the conventional
Al-DSTC. Also, the proposed scheme has a lower bit error rate compared to the beamforming when the transmit power is high. Also, the proposed system has lower BER compared to the conventional Al-DSTC for all transmit power.

We also compare the performance of this schemes based on the power efficiency. We found that the proposed scheme is more efficient than the conventional Al-DSTC and beamforming for all transmit power. Also, the proposed system increases the life time of the relay since $50 \%$ of time the relay is off. This technique can also be extended to multi-antenna relay network.

\section{REFERENCES}

[1] A. Goldsmith, Wireless Communications. New York, NY, USA: Cambridge University Press, 2005.

[2] W. C. Jakes and D. C. Cox, eds., Microwave Mobile Communications. Wiley-IEEE Press, 1994.

[3] G. Pottie, "System design choices in personal communications," Personal Communications, IEEE, vol. 2, no. 5, pp. 50-67, 1995.

[4] C.-E. W. Sundberg and N. Seshadri, "Digital cellular systems for north america," in Global Telecommunications Conference, 1990, and Exhibition. 'Communications: Connecting the Future', GLOBECOM '90., IEEE, pp. 533-537 vol.1, 1990.

[5] P. Balaban and J. Salz, "Dual diversity combining and equalization in digital cellular mobile radio," Vehicular Technology, IEEE Transactions on, vol. 40, no. 2, pp. 342-354, 1991.

[6] J. Winters, J. Salz, and R. Gitlin, "The impact of antenna diversity on the capacity of wireless communication systems," Communications, IEEE Transactions on, vol. 42, no. 234, pp. 1740-1751, 1994.

[7] J. Laneman, D. Tse, and G. W. Wornell, "Cooperative diversity in wireless networks: Efficient protocols and outage behavior," Information Theory, IEEE Transactions on, vol. 50, no. 12, pp. 3062-3080, 2004.

[8] A. Sadek, W. Su, and K. J. R. Liu, "Multinode cooperative communications in wireless networks," Signal Processing, IEEE Transactions on, vol. 55, no. 1, pp. 341-355, 2007.

[9] Z. Yi and I.-M. Kim, "Diversity order analysis of the decode-andforward cooperative networks with relay selection," Wireless Communications, IEEE Transactions on, vol. 7, no. 5, pp. 1792-1799, 2008.

[10] J. Laneman, G. W. Wornell, and D. Tse, "An efficient protocol for realizing cooperative diversity in wireless networks," in Information Theory, 2001. Proceedings. 2001 IEEE International Symposium on, pp. 294-, 2001.

[11] H. Suraweera, D. Michalopoulos, and G. Karagiannidis, "Performance of distributed diversity systems with a single amplify-and-forward relay," Vehicular Technology, IEEE Transactions on, vol. 58, no. 5, pp. 26032608, 2009.

[12] J. Laneman and G. W. Wornell, "Distributed space-time-coded protocols for exploiting cooperative diversity in wireless networks," Information Theory, IEEE Transactions on, vol. 49, no. 10, pp. 2415-2425, 2003.

[13] P. Larsson and H. Rong, "Large-scale cooperative relaying network with optimal coherent combining under aggregate relay power constraints," in WWRF: New Air Interfaces, Relay based Systems and Smart Antennas, 2004. Qc 20120203.

[14] I. Maric and R. Yates, "Bandwidth and power allocation for cooperative strategies in gaussian relay networks," in Signals, Systems and Computers, 2004. Conference Record of the Thirty-Eighth Asilomar Conference on, vol. 2, pp. 1907-1911 Vol.2, 2004.

[15] Z. Fang, Y. Hua, and J. Koshy, "Joint source and relay optimization for a non-regenerative mimo relay," in Sensor Array and Multichannel Processing, 2006. Fourth IEEE Workshop on, pp. 239-243, 2006.

[16] Y. Jing and H. Jafarkhani, "Network beamforming using relays with perfect channel information," Information Theory, IEEE Transactions on, vol. 55, no. 6, pp. 2499-2517, 2009.

[17] Y. Jing and H. Jafarkhani, "Single and multiple relay selection schemes and their achievable diversity orders," Wireless Communications, IEEE Transactions on, vol. 8, no. 3, pp. 1414-1423, 2009.

[18] Y. Jing and H. Jafarkhani, "Using orthogonal and quasi-orthogonal designs in wireless relay networks," Information Theory, IEEE Transactions on, vol. 53, no. 11, pp. 4106-4118, 2007. 
[19] Y. Jing and B. Hassibi, "Distributed space-time coding in wireless relay networks," Wireless Communications, IEEE Transactions on, vol. 5, no. 12 , pp. 3524-3536, 2006.

[20] B. Maham, A. Hjorungnes, and G. Abreu, "Distributed gabba spacetime codes in amplify-and-forward relay networks," Wireless Communications, IEEE Transactions on, vol. 8, no. 4, pp. 2036-2045, 2009.

[21] G. Rajan and B. Rajan, "Distributed space-time codes for cooperative networks with partial csi," in Wireless Communications and Networking Conference, 2007.WCNC 2007. IEEE, pp. 902-906, 2007.

[22] B. Maham and A. Hjorungnes, "Power allocation strategies for distributed space-time codes in amplify-and-forward mode," EURASIP Journal on Advances in Signal Processing, vol. 2009, no. 1, p. 612719, 2009.

[23] D. H. N. Nguyen, H. Nguyen, and H. Tuan, "A novel power allocation scheme for distributed space-time coding," in Communications, 2009. ICC '09. IEEE International Conference on, pp. 1-6, 2009.

[24] Y. Jing and H. Jafarkhani, "Relay power allocation in distributed spacetime coded networks with channel statistical information," Wireless Communications, IEEE Transactions on, vol. 10, no. 2, pp. 443-449, 2011.

[25] D. Gore and A. Paulraj, "Mimo antenna subset selection with spacetime coding," Signal Processing, IEEE Transactions on, vol. 50, no. 10, pp. 2580-2588, 2002.

[26] S. Alamouti, "A simple transmit diversity technique for wireless communications," Selected Areas in Communications, IEEE Journal on, vol. 16, no. 8, pp. 1451-1458, 1998. 